\title{
THE LEVEL MANIFOLD OF A GENERALIZED TODA EQUATION HIERARCHY
}

\author{
YOSHIMASA NAKAMURA
}

\begin{abstract}
The finite nonperiodic Toda lattice equation induces a linear oneparameter flow on a space of rational functions. The level manifold of the Toda equation hierarchy is shown to be a product of lines. Our main results establish a generalization of this Toda hierarchy which will be called the cyclic-Toda hierarchy. It is proved that the cyclic-Toda hierarchy is completely integrable and its level manifold is diffeomorphic to a disjoint union of cylinders.
\end{abstract}

\section{INTRODUCTION}

Let $\mathscr{R} a t(n)$ be a space of real coefficient rational functions of the form

$$
f(z)=\frac{q(z)}{p(z)}=\frac{q_{n-1} z^{n-1}+\cdots+q_{1} z+q_{0}}{z^{n}+p_{n-1} z^{n-1}+\cdots+p_{1} z+p_{0}},
$$

where $\left\{p_{k}, q_{k}\right\}_{k \in \mathcal{N}} \in \mathbb{R}^{2 n}$ with $\mathscr{N}=\{0,1, \ldots, n-1\}, z \in \mathbb{C} \cup\{\infty\}$, and the numerator $q(z)$ and the denominator $p(z)$ do not have any common factor. It is known that each $f(z)$ can be labelled by the Cauchy index $l \in\{-n,-n+$ $2, \ldots, n-2, n\}$, which is a winding number as $z$ ranges over the reals from $-\infty$ to $+\infty[\mathrm{G}]$. Thus the number of the connected components of $\mathscr{R}$ at $(n)$ is $n+1$ [B]. Let $\mathscr{R} a t^{l}(n)$ be the connected component having the Cauchy index $l$. It is to be noted that each rational function $f(z)$ of the component $\mathscr{R} a t^{n}(n)$ admits the partial fraction expansion

$$
f(z)=\frac{1}{\sum_{j \in \mathcal{N}} \exp \left(\alpha_{j}\right)} \sum_{k \in \mathcal{N}} \frac{\exp \left(\alpha_{k}\right)}{z-\zeta_{k}},
$$

where $\left\{\alpha_{k}, \zeta_{k}\right\}_{k \in \mathcal{N}} \in \mathbb{R}^{2 n}$ and $\zeta_{j} \neq \zeta_{k}$ for $j \neq k$.

The finite nonperiodic Toda equation is a completely integrable dynamical system which is of great interest from the point of view of pure mathematics as well as applications. It originated as a description of a one-dimensional lattice of particles with nearest neighbour exponential interaction. The equation of motion takes a Lax form [F] which is a system of nonlinear ODEs. Moser [M]

Received by the editors April 27, 1990 and, in revised form, July 20, 1990. This work was presented October 26, 1989 at the meeting "CRM Workshop on Hamiltonian Systems, Transformation Groups and Spectral Transform Methods" sponsored by Centre de Recherches Mathématiques, Université de Montréal.

1980 Mathematics Subject Classification (1985 Revision). Primary 58F07; Secondary 53C80, $58 \mathrm{~F} 05$. 
showed that the equation of motion can be linearized to the system

$$
\frac{d \alpha_{k}}{d t_{1}}=\zeta_{k}, \quad \frac{d \zeta_{k}}{d t_{1}}=0
$$

for $k \in \mathscr{N}$, where $t_{1} \in \mathbb{R}$ and $\left\{\alpha_{k}, \zeta_{k}\right\}_{k \in \mathcal{N}}$ are coordinates of $\mathscr{R} a t^{n}(n)$. This implies that the Toda equation induces a linear one-parameter flow on $\mathscr{R} a t^{n}(n), \phi_{J_{1}}: \mathbb{R} \times \mathscr{R} a t^{n}(n) \rightarrow \mathscr{R} a t^{n}(n)$, defined by

$$
\begin{aligned}
\phi_{J_{1}} & \left(t_{1}, \frac{1}{\sum_{j \in \mathcal{N}} \exp \left(\alpha_{j}\right)} \sum_{k \in \mathcal{N}} \frac{\exp \left(\alpha_{k}\right)}{z-\zeta_{k}}\right) \\
= & \frac{1}{\sum_{j \in \mathcal{N}} \exp \left(\alpha_{j}+t_{1} \zeta_{j}\right)} \sum_{k \in \mathcal{N}} \frac{\exp \left(\alpha_{k}+t_{1} \zeta_{k}\right)}{z-\zeta_{k}} .
\end{aligned}
$$

Note that the denominator does not change under the action.

For a fixed denominator $p(z)$, we define a subspace

$$
\mathscr{R} a t_{p}(n)=\left\{f(z)=\frac{q(z)}{p(z)} \in \mathscr{R} a t(n) \mid p(z): \text { fixed }\right\}
$$

of $\mathscr{R a t}(n)$. This class of rational functions also appears in control theory as a family of controllable linear dynamical systems with constant coefficients. The number of connected components of $\mathscr{R} a t_{p}(n)$ is $2^{r}$, where $r$ is the number of real distinct roots of $p(z)[\mathrm{KM}]$. Krishnaprasad [K] proved that each connected component can be identified with certain cylinders (product of lines and circles) in terms of the local geometry of $\mathscr{R} a t_{p}(n)$. For example, the connected component $\mathscr{R} a t_{p}^{n}(n)$ of $\mathscr{R} a t_{p}(n)$ having the Cauchy index $n$ is diffeomorphic to $\mathbb{R}^{n}$, where $p(z)=\prod_{k \in \mathcal{N}}\left(z-\zeta_{k}\right),\left\{\zeta_{k}\right\}_{k \in \mathcal{N}} \in \mathbb{R}^{n}$ and $\zeta_{j} \neq \zeta_{k}$ for $j \neq k$. It will be shown in $\S 4$ that $\mathscr{R} a t_{p}^{n}(n)$ can be regarded as the level manifold of the finite nonperiodic Toda equation hierarchy. This explains why the Toda equation induces a one-parameter flow on $\mathscr{R} a t_{p}^{n}(n)$. However it is not known what class of nonlinear integrable systems has level manifold diffeomorphic to the cylinders. This bring us to the main purpose of this paper.

Recently the author $\left[\mathrm{N}_{1}\right]$ considered a space of complex coefficient rational functions which emerges in gauge theory [D]. The space is smooth and connected, and can be regarded as the moduli space of $\mathfrak{S U}(2) n$-monopoles. In $\left[\mathrm{N}_{1}\right]$, a generalized Toda equation called the complex cyclic-Toda hierarchy is found to parametrize the moduli space of monopoles. Physically, the induced flow on the moduli space describes a phase shift of monopoles. A control theoretical picture of the real cyclic-Toda hierarchy has been discussed in $\left[\mathrm{N}_{2}\right]$. A member of the hierarchy describes a time shift of the associated controllable linear dynamical system. Here the notion of hierarchy structure of nonlinear integrable systems has played an important role in mathematical physics. The terminology "hierarchy" denotes a system of (usually infinite) equations describing a commuting set of dynamical flows on some (infinite-dimensional) manifold. In a special sector, the original nonlinear system, such as the KdV equation, is included. Several classes of hierarchies are known, for example, the $\mathrm{KP}$ hierarchy (a generalization of the KdV hierarchy) [SS, DKJM], the infinite Toda hierarchy [UT], and the self-dual Yang-Mills hierarchy [ $\left.\mathrm{N}_{3}, \mathrm{~T}\right]$. In [M], Moser already pointed out a finite hierarchy structure of the finite nonperiodic Toda equation as an analogue of the KdV hierarchy. Moser's hierarchy can be 
viewed as a finite-dimensional sector of the infinite Toda hierarchy and will be called the Jacobi-Toda hierarchy in this paper.

In the next section we introduce the cyclic-Toda hierarchy, which is a compatible nonlinear system of Lax form with supplementary linear systems. This hierarchy describes an isospectral deformation of cyclic matrices and can be regarded as a natural generalization of the Jacobi-Toda hierarchy. In $\S 3$, we construct a unique solution of the cyclic-Toda hierarchy by using the QR decomposition of nonsingular matrices. The Jacobi-Toda hierarchy is an isospectral deformation equation of Jacobi matrices. The level manifold of the Jacobi-Toda hierarchy is shown to be diffeomorphic to $\mathbb{R}^{n}$ in $\S 4$. The cyclic-Toda hierarchy in fact induces a flow on submanifolds of $\mathscr{R} a t_{p}(n)$ having any Cauchy index $l$, as observed in $\S 5$. In $\S 6$, we prove that the cyclic-Toda hierarchy is a completely integrable Hamiltonian system and its level manifold is a disjoint union of cylinders $T^{m} \times \mathbb{R}^{n-m}$. The cyclic-Toda hierarchy would be presumably the first example of a finite-dimensional hierarchy whose level manifold has nontrivial topology.

\section{DEFINITION OF THE CYCLIC-TODA HIERARCHY}

In this section we introduce the real cyclic-Toda hierarchy and show how the hierarchy is related to the usual finite nonperiodic Toda equation. Let $t$ be a set of $n$ real parameters (time variables),

$$
t=\left\{t_{0}, t_{1}, \ldots, t_{n-1}\right\}=\left\{t_{k}\right\}_{k \in \mathscr{N}} \in \mathbb{R}^{n} .
$$

Let $A=A(t)$ be an $n \times n$ matrix function of $t$ satisfying the system of $n$ nonlinear equations of Lax form

$$
\frac{\partial A(t)}{\partial t_{k}}=\left[A^{k}(t)_{\llcorner}^{\top}-A^{k}(t)_{\angle}, A(t)\right]
$$

for $k \in \mathscr{N}$. Here $A_{\angle}$ and $A^{\top}$ denote the strictly lower triangular part and the transpose of $A$, respectively. Obviously, $A(t)$ does not depend on $t_{0}$.

Next we discuss the compatibility of (7). Set

$$
F_{k}(t)=A^{k}(t)^{\top}-A^{k}(t) \angle \cdot
$$

The compatibility conditions lead to the system of zero curvature equations

$$
\frac{\partial F_{k}}{\partial t_{l}}-\frac{\partial F_{l}}{\partial t_{k}}+\left[F_{k}, F_{l}\right]=0
$$

for $k, l \in \mathscr{N}$. Namely, if (8) is satisfied, then (7) is compatible. We can prove the converse of this as follows. By using a similarity action $G: A \rightarrow G A G^{-1}$, where $G$ is a sequence of Householder matrices, we can always assume that the initial value $A(0)$ takes the form of an upper Hessenberg matrix:

$$
\left(\begin{array}{cccc}
* & \ldots & \ldots & * \\
* & \ddots & & \vdots \\
& \ddots & \ddots & \vdots \\
0 & & * & *
\end{array}\right) .
$$

Since (7) is a system of isospectral deformation equations of $A(0)$ (from the standard theory of Lax equations), $\operatorname{Spec} A(t)=\operatorname{Spec} A(0)$. There is a lemma 
in linear algebra [G] which states that $U(t) A(t)=A(0) U(t)$ for some nonsingular upper triangular matrix $U(t)$. This asserts that $A(t)$ also takes the upper Hessenberg form for any $t \in \mathbb{R}^{n}$. Thus $F_{k}$ are skew-symmetric band matrices. From (7),

$$
\frac{\partial A^{l}(t)}{\partial t_{k}}-\left[F_{k}(t), A^{l}(t)\right]=0
$$

for $l \in \mathcal{N}$. Inserting $A^{l}=G_{l}-F_{l}$ into the above, where $G_{l}$ are upper triangular, we can see that $F_{k}$ should satisfy the system of zero curvature equations (8). Namely, the dynamical flows induced by (7) mutually commute. It is shown that (7) is equivalent to (8). The details of the proof can be given similarly to $\left[\mathrm{N}_{1}\right.$, Proposition 1] (for skew-hermitian band matrices).

When $A(t)$ is similar to a Jacobi matrix of the form

$$
A_{J}(t)=\left(\begin{array}{ccccc}
a_{1} & b_{1} & & & 0 \\
b_{1} & a_{2} & \ddots & & \\
& \ddots & \ddots & \ddots & \\
& & \ddots & a_{n-1} & b_{n-1} \\
0 & & & b_{n-1} & a_{n}
\end{array}\right)(t)
$$

with $b_{j}(t)>0$, the compatible system (7) is essentially equivalent to Moser's system of finite nonperiodic Toda equations $[\mathrm{M}]$. He considered the case where $t_{1}=t_{2}=\cdots=t_{n-1}$ and showed that the original Toda equation (with respect to $\left.t_{1}\right)$ induces the flow (4) on $\mathscr{R} a t^{n}(n)$. We recall that each rational function on the flow is expressed as

$$
f\left(z ; t_{1}\right)=e_{1}^{\top}\left(z I-A_{J}\left(t_{1}\right)\right)^{-1} e_{1},
$$

where $e_{1}^{\top}=(10 \cdots 0)$. This class of rational functions always admits the expansion (2) and $\operatorname{det}\left(z I-A_{J}\left(t_{1}\right)\right)$ is independent of $t_{1}$.

Finally, in this section we give additional systems of linear equations. Let $B(t)$ and $C(t)$ be $n \times 1$ and $1 \times n$ vectors, respectively, satisfying

$$
\begin{aligned}
& \frac{\partial B(t)}{\partial t_{k}}=\left(A^{k}(t)+A^{k}(t)_{L^{\top}}-A^{k}(t)_{\angle}\right) B(t), \\
& \frac{\partial C(t)}{\partial t_{k}}=-C(t)\left(A^{k}(t)_{L^{\top}}-A^{k}(t)_{\llcorner}\right)
\end{aligned}
$$

for $k \in \mathscr{N}$. The existence of such $B(t)$ is checked as follows. The compatibility condition of the first equality of $(10)$ is

$$
\frac{\partial A^{k}}{\partial t_{l}}-\left[F_{l}, A^{k}\right]-\frac{\partial A^{l}}{\partial t_{k}}+\left[F_{k}, A^{l}\right]+\frac{\partial F_{k}}{\partial t_{l}}-\frac{\partial F_{l}}{\partial t_{k}}+\left[F_{k}, F_{l}\right]=0
$$

for $k, l \in \mathscr{N}$. Then the compatibility of (7) gives rise to that of the first equality of (10). We omit the compatibility of the second equality.

Now set

$$
\left\{A_{0}, B_{0}, C_{0}\right\}=\{A(0), B(0), C(0)\} .
$$

The vectors $B$ and $C$ are called cyclic vectors of some matrix $A$ if they satisfy

$$
\operatorname{rank}\left(B A B \cdots A^{n-1} B\right)=n, \quad \operatorname{rank}\left(C^{\top} A^{\top} C^{\top} \cdots A^{\top n-1} C^{\top}\right)=n,
$$


respectively. The matrix $A$ is a cyclic matrix of $B$ and $C$. To investigate the level manifold in terms of $\mathscr{R} a t(n)$ we assume that $B_{0}$ and $C_{0}$ are cyclic vectors of some $A_{0}$ in the subsequent sections. Equivalently, we assume that the triplet (11) satisfies for all $\lambda \in \mathbb{C}$

(i) $\left(\lambda I-A_{0} \quad B_{0}\right)$ is surjective,

(ii) $\left(\begin{array}{c}\lambda I-A_{0} \\ C_{0}\end{array}\right)$ is injective.

We shall call the generalized Toda equation hierarchy (7) with the supplementary systems (10) satisfying (i) and (ii) the (real) cyclic-Toda hierarchy. We also call the hierarchy where $A_{0}$ is similar to a Jacobi matrix the Jacobi-Toda hierarchy.

\section{Construction OF SOlutions}

Since the cyclic-Toda hierarchy (7) and (10) satisfies the Lipschitz condition (for any $t \in \mathbb{R}^{n}$ ), there exist a unique solution $\{A(t), B(t), C(t)\}$ for any initial value (11). In this section we construct the solution explicitly by using the $Q R$ decomposition (a Gram-Schmidt orthogonalization procedure). Consider the $\mathrm{QR}$ decomposition of the matrix exponential

$$
\exp \left(\sum_{k \in \mathcal{N}} t_{k} A_{0}^{k}\right)=Q^{-1}(t) R(t),
$$

where $Q$ is an orthogonal matrix and $R$ is an upper triangular matrix with positive diagonal entries. Since $\exp \left(\sum_{k \in \mathcal{N}} t_{k} A_{0}^{k}\right)$ is nonsingular, there uniquely exist the factors such that $Q(0)=I$ and $R(0)=I$. We can prove

Lemma 1. The triplet $\{A(t), B(t), C(t)\}$ defined by

$$
A(t)=Q(t) A_{0} Q^{-1}(t), \quad B(t)=R(t) B_{0}, \quad C(t)=C_{0} Q^{-1}(t)
$$

uniquely solves the initial value problem for (7) and (10) for any $t \in \mathbb{R}^{n}$.

Proof. Taking derivatives of both sides of (12), we have

$$
Q A_{0}^{k} Q^{-1}=-\frac{\partial Q}{\partial t_{k}} \cdot Q^{-1}+\frac{\partial R}{\partial t_{k}} \cdot R^{-1}
$$

for $k \in \mathscr{N}$. Since the first and the second terms of the r.h.s. are skew symmetric and upper triangular, respectively, it follows that

$$
\begin{aligned}
\frac{\partial Q}{\partial t_{k}} \cdot Q^{-1} & =\left(Q A_{0}^{k} Q^{-1}\right) \angle^{\top}-\left(Q A_{0}^{k} Q^{-1}\right) \angle=A^{k}(t)^{\top}-A^{k}(t) \angle, \\
\frac{\partial R}{\partial t_{k}} \cdot R^{-1} & =Q A_{0}^{k} Q^{-1}+\left(Q A_{0}^{k} Q^{-1}\right) \angle^{\top}-\left(Q A_{0}^{k} Q^{-1}\right) \angle \\
& =A^{k}(t)+A^{k}(t) \angle^{\top}-A^{k}(t) \angle .
\end{aligned}
$$

On the other hand, taking derivatives of (13), we have

$$
\begin{aligned}
& \frac{\partial A(t)}{\partial t_{k}}=\left[\frac{\partial Q}{\partial t_{k}} \cdot Q^{-1}, A(t)\right], \\
& \frac{\partial B(t)}{\partial t_{k}}=\frac{\partial R}{\partial t_{k}} \cdot R^{-1} B(t), \quad \frac{\partial C(t)}{\partial t_{k}}=-C(t) \frac{\partial Q}{\partial t_{k}} \cdot Q^{-1} .
\end{aligned}
$$

We can easily check that $\{A(t), B(t), C(t)\}$ given by (12) and (13) satisfies (7) and $(10)$ and $\{A(0), B(0), C(0)\}=\left\{A_{0}, B_{0}, C_{0}\right\}$. 
We obtain a nontrivial flow $\left\{A_{0}, B_{0}, C_{0}\right\} \rightarrow\{A(t), B(t), C(t)\}$ in $\mathbb{R}^{n \times n+2 n}$ from Lemma 1. The solution $A(t)$ is orthogonally similar to $A_{0}$. Thus the eigenvalues of $A_{0}$ clearly give constants of motion of (7). The evolution (13) is a continuous time version $\left[\mathrm{N}_{2}\right]$ of the $\mathrm{QR}$ algorithm for finding eigenvalues of Hessenberg matrices. It was Symes' observation [S] that the QR algorithm of Jacobi matrices is closely linked to the integration of the Jacobi-Toda equation. Recently Moser and Veselov [MV] showed that by factoring quadratic, in a parameter, matrix polynomials and by exchanging the factors a class of discrete analogues of the Lax equations can be integrated.

\section{THE LEVEL MANIFOLD OF THE JACOBI-TODA HIERARCHY}

In the Jacobi case the eigenvalues $\zeta_{k}$ of $A_{0}$ are real distinct. The finite nonperiodic Toda equation itself is a Hamiltonian system with the Hamiltonian

$$
H_{J}=\frac{1}{2} \operatorname{trace} A_{J}^{2} \text {. }
$$

The Toda equation induces the flow (4) on $\mathscr{R} a t^{n}(n)$. We remark that the 2-form

$$
\omega_{J}=\sum_{k \in \mathscr{N}} d \alpha_{k} \wedge d \zeta_{k}
$$

defines a symplectic structure on $\mathscr{R} a t^{n}(n)$. The 2 -form $\omega_{J}$ is a slight modification of that given in [DLuTr]. Let us consider the level manifold of the Jacobi-Toda hierarchy. Set

$$
H_{j}=\frac{1}{j+1} \operatorname{trace} A_{J}^{j+1}(t)
$$

for $j \in \mathscr{N}$. From (7), we can easily check that

$$
\frac{\partial H_{j}}{\partial t_{k}}=0
$$

for $j, k \in \mathscr{N}$. This implies that the $H_{j}$ are constants of motion. Indeed, we can write down $H_{j}$ explicitly, $H_{j}=\frac{1}{j+1} \sum_{k \in \mathcal{N}} \zeta_{k}^{j+1}$. Define the Hamiltonian vector fields on $\mathscr{R} a t^{n}(n)$ by

$$
X_{j}=\sum_{k \in \mathscr{N}} \zeta_{k}^{j} \frac{\partial}{\partial \alpha_{k}}
$$

for $j \in \mathcal{N}$. Clearly $\left[X_{j}, X_{k}\right]=0$, namely, $H_{j}$ are in involution. The level manifold is now defined by

$$
\mathscr{M}_{J}=\left\{f(z) \in \mathscr{R} a t_{p}^{n}(n) \mid H_{j}=c_{j}, j \in \mathscr{N}\right\},
$$

where $\mathscr{R} a t_{p}^{n}(n)=\mathscr{R} a t^{n}(n) \cap \mathscr{R} a t_{p}(n)$ and $c_{j}$ are constants. Clearly, $\mathscr{M}_{J}$ is a connected submanifold of $\mathscr{R} a t^{n}(n)$. From (14) we have

Lemma 2. The vector fields $X_{j}$ are complete and act transitively on the level manifold $\mathscr{M}_{J}$.

Furthermore, the action of $\left\{X_{j}\right\}_{j \in \mathcal{N}}$ is free and can be expressed as $\phi_{J}$ : 
$\mathbb{R}^{n} \times \mathscr{M}_{J} \rightarrow \mathscr{M}_{J}$ defined by

$$
\begin{aligned}
\phi_{J}(t & \left., \frac{1}{\sum_{j \in \mathscr{N}} \exp \left(\alpha_{j}\right)} \sum_{k \in \mathcal{N}} \frac{\exp \left(\alpha_{k}\right)}{z-\zeta_{k}}\right) \\
& =\frac{1}{\sum_{j \in \mathscr{N}} \exp \left(\alpha_{j}+\sum_{l \in \mathcal{N}} t_{l} \zeta_{k}^{l}\right)} \sum_{k \in \mathcal{N}} \frac{\exp \left(\alpha_{k}+\sum_{j \in \mathcal{N}} t_{j} \zeta_{k}^{j}\right)}{z-\zeta_{k}} .
\end{aligned}
$$

It has been proved that

Proposition 1. The level manifold $\mathscr{M}_{J}$ of the Jacobi-Toda hierarchy is diffeomorphic to the product of $n$-lines, that is, $\mathscr{M}_{J} \simeq \mathbb{R}^{n}$.

As a consequence of the above discussion we have the following (with obvious proof)

Proposition 2. The Jacobi-Toda hierarchy is linearized on $\mathscr{R} a t^{n}(n)$ to

$$
\frac{\partial \alpha_{k}}{\partial t_{j}}=\zeta_{k}^{j}, \quad \frac{\partial \zeta_{k}}{\partial t_{j}}=0
$$

for all $j, k \in \mathscr{N}$.

Compare (17) with Moser's system (3). The coordinates $\zeta_{k}$ and $\alpha_{k}$ are regarded as the action and angle variables, respectively. We have shown that the Jacobi-Toda hierarchy induces a linear $n$-parameter flow on $\mathscr{R} a t^{n}(n)$. Krishnaprasad [K] defined the level manifold $\mathscr{M}_{J}^{\prime}$ of the linearlized JacobiToda equation (3) by

$$
\mathscr{M}_{J}^{\prime}=\left\{f(z) \in \mathscr{R} a t_{p}^{n}(n) \mid \zeta_{j}=c_{j}^{\prime}, j \in \mathscr{N}\right\}
$$

and gave a proof that $\mathscr{M}_{J}^{\prime} \simeq \mathbb{R}^{n}$ under the assumption that not one of the $c_{j}^{\prime}$ is zero. However, his expression of action is not correct and if one of the $c_{j}^{\prime}$ is zero, then the transitivity is destroyed.

Let us consider the foliation of $\mathscr{R} a t^{n}(n)$,

$$
\mathscr{R} a t^{n}(n)=\bigcup_{p \in \mathbb{R}^{n}} \mathscr{R} a t_{p}^{n}(n),
$$

where $p=\left\{p_{k}\right\}_{k \in \mathscr{N}}$ with $p(z)=\prod_{k \in \mathbb{N}}\left(z-\zeta_{k}\right)$. Since the action $\phi_{J}$ does not change $\left\{\zeta_{k}\right\}_{k \in \mathscr{N}}$, the level manifold $\mathscr{M}_{J}$ can be identified with the leaf $\mathscr{R} a t_{p}^{n}(n)$.

\section{THE CYCLIC-TODA HIERARCHY AND RATIONAL FUNCTIONS}

We first fix the expression of the initial value $\left\{A_{0}, B_{0}, C_{0}\right\}$ in a canonical form in order to carry out a one-to-one correspondence between each triplet and rational function of $\mathscr{R} a t(n)$. For any $\left\{A_{0}, B_{0}, C_{0}\right\}$, define

$$
f(z ; 0)=C_{0}\left(z I-A_{0}\right)^{-1} B_{0} .
$$

Since $\left\{A_{0}, B_{0}, C_{0}\right\}$ satisfies (i) and (ii), the degree of $f(z ; 0)$ is precisely $n$. Namely, $f(z ; 0)$ takes the form of $(1)$, where $p(z)$ and $q(z)$ are coprime. Let $\mathscr{C}_{n}(\mathbb{R})$ be a space of cyclic matrices and vectors

$$
\mathscr{C}_{n}(\mathbb{R})=\left\{\{A, B, C\} \in \mathbb{R}^{n \times n} \times \mathbb{R}^{n \times 1} \times \mathbb{R}^{1 \times n} \mid B \text { and } C \text { are cyclic vectors of } A\right\} .
$$


Define an algebraic group action on $\mathscr{C}_{n}(\mathbb{R}), \beta: \mathfrak{G} \mathfrak{L}(n, \mathbb{R}) \times \mathscr{C}_{n}(\mathbb{R}) \rightarrow \mathscr{C}_{n}(\mathbb{R})$, by

$$
\beta(G, A, B, C)=\left(G A G^{-1}, G B, C G^{-1}\right) \text {. }
$$

Let us call $\beta$ the similarity action on $\mathscr{C}_{n}(\mathbb{R})$. It is to be noted that if $\left\{A_{0}, B_{0}\right.$, $\left.C_{0}\right\}$ gives $f(z ; 0)$ through (18), then so does $\left\{G A_{0} G^{-1}, G B_{0}, C_{0} G^{-1}\right\}$ for any $\mathfrak{G} \mathfrak{L}(n, \mathbb{R})$ matrix $G$. Hence the orbit space $\mathscr{C}_{n}(\mathbb{R}) / \mathfrak{G} \mathfrak{L}(n, \mathbb{R})$ of the similarity action can be viewed as the space $\mathscr{R} a t(n)$. Using $\beta$, for any given rational function $f(z ; 0)$ we can uniquely determine a matrix $A_{0}$ and its cyclic vectors $B_{0}$ and $C_{0}$ of the form

$$
A_{0}=\left(\begin{array}{cccc}
0 & & 0 & -p_{0} \\
1 & \ddots & & \vdots \\
& \ddots & 0 & -p_{n-2} \\
0 & & 1 & -p_{n-1}
\end{array}\right), \quad B_{0}=\left(\begin{array}{c}
q_{0} \\
\vdots \\
q_{n-2} \\
q_{n-1}
\end{array}\right), \quad C_{0}=\left(\begin{array}{llll}
0 & \cdots & 0 & 1
\end{array}\right),
$$

and vice versa. The following proposition is the key connection between the level manifold and the space of rational functions.

Proposition 3. If $B_{0}$ and $C_{0}$ are cyclic vectors of $A_{0}$, then $B(t)$ and $C(t)$ are cyclic vectors of $A(t)$ for any $t \in \mathbb{R}^{n}$.

Proof. Consider the $\mathfrak{G} \mathfrak{L}(n, \mathbb{R})$ similarity action $\beta$ induced by $G=R(t)$ to get

$$
\operatorname{rank}\left(\lambda I-A_{0} B_{0}\right)=\operatorname{rank}\left(\lambda I-R(t) A_{0} R^{-1}(t) R(t) B_{0}\right) .
$$

From Lemma 1 we see that $\left(\lambda I-R A_{0} R^{-1} R B_{0}\right)=\left(\lambda I-R Q^{-1} A Q R^{-1} B\right)$. Here let us note that $R Q^{-1}=Q \exp \left(A_{0}\right) Q^{-1}=\exp (A)$ from (12). Thus it is proved that

$$
\operatorname{rank}\left(\lambda I-A_{0} B_{0}\right)=\operatorname{rank}(\lambda I-A(t) B(t)) .
$$

On the other hand, using the similarity action induced by $G=Q(t)$ and Lemma 1 we have

$$
\operatorname{rank}\left(\begin{array}{c}
\lambda I-A_{0} \\
C_{0}
\end{array}\right)=\operatorname{rank}\left(\begin{array}{c}
\lambda I-Q(t) A_{0} Q^{-1}(t) \\
C_{0} Q^{-1}(t)
\end{array}\right)=\operatorname{rank}\left(\begin{array}{c}
\lambda I-A(t) \\
C(t)
\end{array}\right) .
$$

From the assumption we check that $\{A(t), B(t), C(t)\}$ actually satisfies (i) and (ii) for any $t \in \mathbb{R}^{n}$.

Corollary 1. If $f(z ; 0) \in \mathscr{R} a t^{l}(n)$, then the parametric rational function defined by

$$
f(z ; t)=C(t)(z I-A(t))^{-1} B(t)
$$

is an element of $\mathscr{R} a t^{l}(n)$ for any $t \in \mathbb{R}^{n}$.

This implies that the cyclic-Toda hierarchy induces a flow on each connected component $\mathscr{R} a t^{l}(n)$ determined by choice of the initial value $\left\{A_{0}, B_{0}, C_{0}\right\}$. Moreover, since $\operatorname{det}(z I-A(t))=\operatorname{det}\left(z I-A_{0}\right)$, we have

Proposition 4. If $f(z ; 0) \in \mathscr{R} a t_{p}(n)$, then $f(z ; t) \in \mathscr{R} a t_{p}(n)$ for any $t \in \mathbb{R}^{n}$.

The space $\mathscr{R} a t_{p}(n)$ has $2^{r}$ connected components, where $r$ is the number of real distinct roots $\zeta_{j}$ of $p(z)$. Write $\zeta_{j}$ in order, $\zeta_{1}<\zeta_{2}<\cdots<\zeta_{r}$. Let $s$ be a multi-index $s=\left\{s_{1}, s_{2}, \ldots, s_{r}\right\}$, where $s_{j}= \pm 1$. If an odd number 
of zeros of the numerator $q(z)$ are on the interval $\left(\zeta_{j}, \zeta_{j+1}\right)$, we assign 1 to $s_{j}$ for $1 \leq j \leq r-1$. Otherwise, we assign -1 to $s_{j}$. Set $s_{r}=1$ if the coefficient of the highest power of $z$ in $q(z)$ is positive. Otherwise, set $s_{r}=-1$. Now each connected component of $\mathscr{R} a t_{p}^{l}(n)=\mathscr{R} a t^{l}(n) \cap \mathscr{R} a t_{p}(n)$ can be distinguished by the index $s$. In what follows let $\mathscr{R} a t_{p}^{l, s}(n)$ denote each connected component. Consequently, we have

$$
\mathscr{R} a t_{p}(n)=\bigcup_{l} \bigcup_{s} \mathscr{R} a t_{p}^{l, s}(n) \text {. }
$$

The index $s$ depends on the Cauchy index $l$. For example, if

$$
p(z)=\prod_{k \in \mathscr{N}}\left(z-\zeta_{k}\right) \quad \text { for } \zeta_{k} \neq \zeta_{l}, \zeta_{k} \in \mathbb{R},
$$

then $\mathscr{R} a t_{p}^{n}(n)$ itself is connected and is congruent to $\mathscr{R} a t_{p}^{n, s}(n)$ with $s=$ $\{1, \ldots, 1\}$. Moreover, $\mathscr{R} a t_{p}^{-n}=\mathscr{R} a t_{p}^{-n, s^{\prime}}(n)$ with $s^{\prime}=\{-1, \ldots,-1\}$. Taking account of Corollary 1 and Proposition 4 we see that the cyclic-Toda hierarchy induces a flow on $\mathscr{R} a t_{p}^{l, s}(n)$.

\section{THE LEVEL MANIFOLD OF THE CYCLIC-TODA HIERARCHY}

First we show the complete integrability of the cyclic-Toda hierarchy simply by letting the cyclic matrix $A(t)$ give rise to constants of motion.

Define

$$
H_{j}=\frac{1}{j+1} \operatorname{trace} A^{j+1}(t)
$$

for $j \in \mathscr{N}$. From (7) we see that

$$
\frac{\partial H_{j}}{\partial t_{k}}=0
$$

for $j, k \in \mathscr{N}$, namely, the $H_{j}$ are constants of motion. Let us introduce the level manifold $\mathscr{M}_{c}$ of the cyclic-Toda hierarchy through

$$
\mathscr{M}_{c}=\bigcup_{l} \bigcup_{s} \mathscr{M}_{c}^{l, s}, \quad \mathscr{M}_{c}^{l, s}=\left\{f(z) \in \mathscr{R} a t_{p}^{l, s}(n) \mid H_{j}=c_{j}, j \in \mathscr{N}\right\},
$$

where $c_{j}$ are constants determined by the coefficients of $p(z)=\operatorname{det}(z I-A)$. Clearly, $\mathscr{M}_{c}$ is not connected and $\mathscr{M}_{c}^{l, s}$ are smooth open submanifolds of $\mathscr{R} a t_{p}^{l, s}(n)$, respectively. From (12) and (13) we get

Lemma 3. The rational function $f(z ; t)$ defined by (20) satisfies

$$
f(z ; t)=C_{0}\left(z I-A_{0}\right)^{-1} \exp \left(\sum_{k \in \mathscr{N}} t_{k} A_{0}{ }^{k}\right) B_{0} .
$$

We choose here local coordinates $\left\{p_{j}, q_{j}\right\}_{j \in \mathscr{N}}$ on $\mathscr{R} a t^{l}(n)$. The Hamiltonian $H_{c}$ of the cyclic-Toda hierarchy is given by $H_{2}$. Consider complete Hamiltonian vector fields on $\mathscr{R} a t^{l}(n)$,

$$
X_{k}=\sum_{i, j=1}^{n} a_{i j}^{k} q_{j-1} \frac{\partial}{\partial q_{i-1}}
$$

for $k \in \mathscr{N}$, where $a_{i j}^{k}$ are the $(i, j)$ elements of $A_{0}^{k}$. It is not hard to see that $\left[X_{j}, X_{k}\right]=0$ for $j, k \in \mathscr{N}$. Since $A_{0}$ is a cyclic matrix, the vector fields are linearly independent. Thus we have 
Theorem 1. The cyclic-Toda hierarchy is completely integrable in the classical sense of Liouville.

In [DLNT], it was shown that the finite nonperiodic Toda equation on a generic orbit (a truncation of Toda equation) is completely integrable. We remark here that every cyclic-Toda hierarchy is not on the generic orbit in their sense. In [DLT] it was shown that the QR algorithm is the integer time evaluation of a completely integrable Hamiltonian system for initial data given by generic matrices. It would be interesting to make clear the relationship between Theorem 1 and the results in [DLNT, DLT].

Now we can show that the level manifold is diffeomorphic to a disjoint union of cylinders. Corollary 1 and Proposition 4 again assert that $\left\{X_{k}\right\}_{k \in \mathcal{N}}$ spans an $n$-dimensional tangent space of $\mathscr{R} a t_{p}^{l, s}(n)$ at any point. Lemma 3 shows that $X_{k}$ act transitively on $\mathscr{M}_{c}^{l, s}$. The action of $\left\{X_{k}\right\}_{k \in \mathcal{N}}$ is abelian and expressed as $\phi_{c}: \mathbb{R}^{n} \times \mathscr{M}_{c}^{l, s} \rightarrow \mathscr{M}_{c}^{l, s}$ defined by

$$
\phi_{c}(t, f(z ; 0))=f(z ; t) .
$$

Hence

$$
\mathscr{M}_{c}^{l, s}=\left\{f(z ; t) \mid f(z ; 0) \in \mathscr{R} a t_{p}^{l, s}(n), t \in \mathbb{R}^{n}\right\} .
$$

Noting that $\operatorname{dim} \mathscr{M}_{c}^{l, s}=n$ and $\mathscr{M}_{c}^{l, s}$ is open, we have

$$
\mathscr{M}_{c}^{l, s}=\mathscr{R}^{l} t_{p}^{l, s}(n),
$$

and consequently, $\mathscr{M}_{c}=\mathscr{R} a t_{p}(n)$.

From the connectedness of $\mathscr{M}_{c}^{l, s}$ we can see that the action $\phi_{c}$ on $\mathscr{M}_{c}^{l, s}$ is free. Let $\Gamma_{f}$ be an isotropy group at any $f(z ; 0) \in \mathscr{R} a t_{p}^{l, s}(n)$ of the abelian action $\phi_{c}$

$$
\Gamma_{f}=\left\{t \in \mathbb{R}^{n} \mid \phi_{c}(t, f(z ; 0))=f(z ; 0)\right\} .
$$

Since $\phi_{c}$ is transitive, the connected component $\mathscr{M}_{c}^{l, s}$ of the level manifold $\mathscr{M}_{c}$ is diffeomorphic to the quotient manifold $\mathbb{R}^{n} / \Gamma_{f}$. Noting again that the $X_{k}$ are linearly independent on $\mathscr{M}_{c}^{l, s}$, we see that $\Gamma_{f}$ is a discrete subgroup of $\mathbb{R}^{n}$. It is known that the discrete subgroup is isomorphic to $\mathbb{Z}^{m}$ for some $m \in \mathscr{N}$. Thus $\mathscr{M}_{c}^{l, s} \simeq \mathbb{R}^{k} / \mathbb{Z}^{m}$. The uniformly varying global coordinates $\psi \bmod 2 \pi$ and $\alpha$ can be expressed as linear functions of $t=\left\{t_{0}, t_{1}, \ldots, t_{n-1}\right\} \in \mathbb{R}^{n}$. Following a theorem in [AKN, p. 110] we have proved

Theorem 2. Each connected component $\mathscr{M}_{c}^{l, s}$ of the level manifold $\mathscr{M}_{c}$ of the cyclic-Toda hierarchy is diffeomorphic to the cylinder $\mathbb{R}^{n} / \mathbb{Z}^{m}=T^{m} \times \mathbb{R}^{n-m}$ for some $m \in \mathcal{N}$. There are coordinates $\psi_{0}, \ldots, \psi_{m-1} \bmod 2 \pi$ and $\alpha_{0}, \ldots$, $\alpha_{n-m-1}$ on $T^{m} \times \mathbb{R}^{n-m}$ in which the cyclic-Toda hierarchy takes the form

$$
\frac{\partial \psi_{h}}{\partial t_{j}}=\omega_{h j}, \quad \frac{\partial \omega_{h j}}{\partial t_{j}}=0, \quad \frac{\partial \alpha_{k}}{\partial t_{j}}=\zeta_{k j}, \quad \frac{\partial \zeta_{k j}}{\partial t_{j}}=0,
$$

where $h \in\{0,1, \ldots, m-1\}, k \in\{0,1, \ldots, n-m-1\}$, and $j \in \mathscr{N}$.

This is in agreement with the Arnold-Liouville theorem in classical mechanics. We recall here the early observation by Krishnaprasad $[\mathrm{K}]$ that $\mathscr{R} a t_{p}^{l, s}(n)$ is diffeomorphic to certain cylinders. Compare (27) with the linearization (17) 
of the Jacobi-Toda hierarchy. Theorem 2 implies that the level manifold $\mathscr{M}_{c}$ is diffeomorphic to the disjoint union (22) of cylinders. In particular, $\mathscr{M}_{c}^{n, s}$ $\left(\simeq \mathbb{R}^{n}\right.$ ) is the level manifold of the Jacobi-Toda hierarchy originally discussed by Moser [M]. Hence we have established a natural generalization of the celebrated finite nonperiodic one-dimensional Toda equation to the cyclic-Toda hierarchy whose level manifold has nontrivial topology. Other types of generalizations have been considered by many authors, based upon the representation theory of Lie algebras (see the recent work [I] and references therein). It should be remarked that the nontrivial topology of the Jacobi-Toda equation was discussed in [To], where the condition $b_{j}(t)>0$ in $A_{J}(t)$ of $\S 2$ is relaxed into $b_{j}(t) \geq 0$. The resulting level manifold is homeomorphic to a convex set in $\mathbb{R}^{n}$ for any fixed set of mutually distinct eigenvalues $\left\{\zeta_{k}\right\}_{k \in \mathscr{N}}$.

\section{Conclusion}

We have a very explicit way of seeing the level manifold of the cyclic-Toda hierarchy which is a new completely integrable dynamical system of finite dimensions. We remark also that the hierarchy may be seen to describe a nonlinear lattice of particles with a long range interaction. As we have observed, the hierarchy completely parametrizes the space $\mathscr{R} a t(n)$ of rational functions. We hope to have demonstrated in this paper that the rich structure of the cyclicToda hierarchy stands revealed.

\section{ACKNOWLEDGMENT}

The author would like to thank J. P. Harnad, T. Iwai, J. K. Moser, A. Tsuchiya, and K. Ueno for stimulating discussions.

\section{REFERENCES}

[AKN] V. I. Arnold, V. V. Kozlov, and A.I. Neishtadt, Mathematical aspects of classical and celestial mechanics, Dynamical Systems III (V. I. Arnold, ed.), Encyclopedia of Math. Sci., vol. 3, Springer-Verlag, Berlin, 1988.

[B] R. W. Brockett, Some geometrical questions in the theory of systems, IEEE Trans. Automat. Control 21 (1976), 449-455.

[DKJM] E. Date, M. Kashiwara, M. Jimbo, and T. Miwa, Transformation groups for soliton equations, Non-Linear Integrable Systems-Classical Theory and Quantum Theory (M. Jimbo and T. Miwa, eds.), World Scientific, Singapore, 1983, pp. 39-119.

[DLNT] P. Deift, L. C. Li, T. Nanda, and C. Tomei, The Toda flow on a generic orbit is integrable, Comm. Pure Appl. Math. 39 (1986), 183-232.

[DLT] P. Deift, L. C. Li, and C. Tomei, Matrix factorizations and integrable systems, Comm. Pure Appl. Math. 42 (1989), 443-521.

[DLuTr] P. Deift, F. Lund, and E. Trobowitz, Nonlinear wave equations and constrained harmonic motion, Comm. Math. Phys. 74 (1980), 141-188.

[D] S. K. Donaldson, Nahm's equations and the classification of monopoles, Commun. Math. Phys. 96 (1984), 387-407.

[F] H. Flaschka, The Toda lattice. I. Existence of integrals, Phys. Rev. D 9 (1974), 1924-1925.

[G] F. R. Gantmacher, The theory of matrices, Chelsea, New York, 1959.

[I] V. I. Inozemtsev, The finite Toda lattices, Comm. Math. Phys. 121 (1989), 629-638.

[K] P. S. Krishnaprasad, Symplectic mechanics and rational functions, Richerche Automat. 10 (1979), 107-135. 
[KM] P. S. Krishnaprasad and C. F. Martin, On families of systems and deformations, Internat. J. Control 38 (1983), 1055-1079.

[M] J. Moser, Finitely many points on the line under the influence of an exponential potential-An integrable system, Dynamical Systems, Theory and Applications (J. Moser, ed.), Lecture Notes in Phys., vol. 38, Springer-Verlag, Berlin, 1975, pp. 467-497.

[MV] J. Moser and A. P. Veselov, Discrete versions of some classical integrable systems and factorization of matrix polynomials, Comm. Math. Phys. 139 (1991), 217-243.

$\left[\mathrm{N}_{1}\right] \quad \mathrm{Y}$. Nakamura, Moduli space of SU(2) monopoles and the complex cyclic-Toda hierarchy, Comm. Math. Phys. 128 (1990), 509-520.

$\left[\mathrm{N}_{2}\right] \quad$, Geometry of rational functions and nonlinear integrable systems, SIAM J. Math. Anal. 22 (1991), 1744-1754.

[ $\left.\mathrm{N}_{3}\right] \quad$, Transformation group acting on a self-dual Yang-Mills hierarchy, J. Math. Phys. 29 (1988), 244-248.

[SS] M. Sato and Y. Sato, Soliton equations as dynamical systems on infinite dimensional Grassmann manifold, Nonlinear PDE in Applied Science (Proc. U.S.-Japan Seminar, Tokyo 1982) (H. Fujita, P. D. Lax, and G. Strang, eds.), Lecture Notes Numer. Appl. Anal., vol. 5, Kinokuniya, Tokyo, 1983, pp. 259-271.

[S] W. W. Symes, The QR algorithm and scattering for the finite nonperiodic Toda lattice, Phys. D 4 (1982), 275-280.

[T] K. Takasaki, Hierarchy structure in integrable systems of gauge fields and underlying Lie algebras, Comm. Math. Phys. 127 (1990), 225-238.

[To] C. Tomei, The topology of isospectral manifolds of tridiagonal matrices, Duke Math. J. 51 (1984), 981-996.

[UT] K. Ueno and K. Takasaki, Toda lattice hierarchy, Group Representations and Systems of Differential Equations (K. Okamoto, ed.), Adv. Stud. in Pure Math. vol. 4, Kinokuniya, Tokyo, 1984, pp. 1-95.

Department of Mathematics, Gifu University, Yanagido, Gifu 501-11, Japan 